\title{
Identification of Biologically Relevant Compounds in Aboveground and Belowground Induced Volatile Blends
}

\author{
Nicole M. van Dam • Bao-Li Qiu • Cornelis A. Hordijk • \\ Louise E. M. Vet • Jeroen J. Jansen
}

Received: 9 June 2010 /Revised: 18 July 2010 / Accepted: 29 July 2010 /Published online: 25 August 2010

(C) The Author(s) 2010. This article is published with open access at Springerlink.com

\begin{abstract}
Plants under attack by aboveground herbivores emit complex blends of volatile organic compounds (VOCs). Specific compounds in these blends are used by parasitic wasps to find their hosts. Belowground induction causes shifts in the composition of aboveground induced VOC blends, which affect the preference of parasitic wasps. To identify which of the many volatiles in the complex VOC blends may explain parasitoid preference poses a challenge to ecologists. Here, we present a case study in which we use a novel bioinformatics approach to identify biologically relevant differences between VOC blends of feral cabbage (Brassica oleracea L.). The plants were induced aboveground or belowground with jasmonic acid (JA) and shoot feeding caterpillars (Pieris brassicae or $P$.
\end{abstract}

\footnotetext{
N. M. van Dam $(\bowtie)$

Radboud University Nijmegen, Institute for Water and Wetland Research (IWWR),

PO Box 9010, 6500 GL Nijmegen, The Netherlands

e-mail: n.vandam@science.ru.nl

C. A. Hordijk - L. E. M. Vet

Netherlands Institute of Ecology (NIOO-KNAW),

PO Box 40, 6666 ZG Heteren, The Netherlands

B.-L. Qiu

Department of Entomology, South China Agricultural University,

510640 Guangzhou, People's Republic of China

\section{E. M. Vet}

Laboratory of Entomology,

Wageningen University and Research Center,

P.O. Box 8031, 6700 EH Wageningen, The Netherlands

\section{J. J. Jansen}

Biosystems Data Analysis, Swammerdam Institute for Lifes

Siences (SILS), University of Amsterdam (UvA),

Nieuwe Achtergracht 166,

1018 WV Amsterdam, The Netherlands

rapae). We used Partial Least Squares-Discriminant Analysis (PLSDA) to integrate and visualize the relation between plant-emitted VOCs and the preference of female Cotesia glomerata. Overall, female wasps preferred JAinduced plants over controls, but they strongly preferred aboveground JA-induced plants over belowground JAinduced plants. PLSDA revealed that the emission of several monoterpenes was enhanced similarly in all JAtreated plants, whereas homoterpenes and sesquiterpenes increased exclusively in aboveground JA-induced plants. Wasps may use the ratio between these two classes of terpenes to discriminate between aboveground and belowground induced plants. Additionally, it shows that aboveground applied JA induces different VOC biosynthetic pathways than JA applied to the root. Our bioinformatic approach, thus, successfully identified which VOCs matched the preferences of the wasps in the various choice tests. Additionally, the analysis generated novel hypotheses about the role of JA as a signaling compound in aboveground and belowground induced responses in plants.

Key Words Aboveground-belowground interactions . Volatile organic compounds · Indirect defenses . Multivariate analysis - Terpenes · Jasmonic acid . Parasitoid preference $\cdot$ PLSDA

\section{Introduction}

It has long been known that pants attacked by aboveground herbivorous insects emit complex blends of volatile organic compounds (VOCs) that are used by predators and parasitic wasps searching for their hosts (Dicke and Sabelis, 1988; Turlings et al., 1990; Geervliet et al., 1997). Only recently has it been discovered that also belowground herbivores 
elicit specific VOCs that attract belowground natural enemies to the roots (Rasmann et al., 2005). Both aboveground and belowground emitted VOCs can act as indirect defenses, attracting natural enemies that eventually kill the herbivore. Aboveground and belowground induced responses, however, are not mutually independent. For example, signaling hormones induced in one compartment may be transported throughout the plant and trigger systemic responses in the undamaged plant parts as well (Bezemer and van Dam, 2005). Recent studies show that root herbivory alters VOC profiles induced by aboveground herbivores feeding on the same plant, which affects the preference of aboveground parasitic wasps searching for aboveground feeding hosts (Rasmann and Turlings, 2007; Soler et al., 2007). These examples illustrate the importance of belowground induced responses for aboveground multitrophic interactions associated with plants.

When VOC blends contain relatively few compounds or when belowground feeding causes a general repression of VOC emissions - as is the case for maize (Rasmann and Turlings, 2007) — the differences between aboveground and belowground induced plants are relatively easily identified. However, in plant species such as Nicotiana attenuata and several Brassicaceous species, which emit complex VOC profiles that contain several hundreds of different individual compounds belonging to various biosynthetic groups, pinpointing shifts in VOC blends is more challenging (van Dam and Poppy, 2008; Bruinsma et al., 2009; Gaquerel et al., 2009). The analytical challenge in detecting shifts in these VOC blends goes beyond detecting a single 'responsible' compound. VOCs, like all metabolites, are produced via intricate biosynthetic networks in which the production of various compounds is interrelated. Another complicating factor is that damage by belowground or aboveground herbivores may cause several VOCs in the profile to change in different directions (Soler et al., 2007; Bruinsma et al., 2009). As 'pure chemicals are rare in nature' and 'real odors are mixtures of volatiles' (Bargmann, 2006), it is seldom that single VOCs are associated with the complete behavioral response of an organism; it is more likely that multiple compounds in the plant-emitted VOC blends serve as cues. Moreover, different compounds in the blend may elicit similar responses, and a single compound may elicit only a behavioral response when offered in the proper background of other plant VOCs (Mumm and Hilker, 2005). Under such conditions, a comprehensive and system-wide approach is needed to identify the biosynthetic shifts that occur in these complex blends, especially when the aim is to correlate multiple changes in VOC blends to binary parasitoid preference tests. Traditional statistical approaches, such as series of ANOVAs on each individual compound, do not provide this comprehensive overview. Therefore, novel bioinformatic approaches based on multivariate data analysis are required to characterize these complex VOC data sets, and link the outcome to ecological data such as preference tests (van Dam and Poppy, 2008).

Multivariate approaches have been used widely in plant metabolomics studies. Only recently are they more commonly implemented for the (unsupervised) analysis of large VOC data sets (Leitner et al., 2008; van Dam and Poppy, 2008; Bruinsma et al., 2009; Gaquerel et al., 2009). Multivariate analyses are tailored to deal with complex data sets that contain variables that are correlated. Interrelated variables are also common to VOC data sets, because they contain groups of VOCs derived from communal biosynthetic pathways, and even from single enzymes producing a range of products (e.g., terpene biosynthetic enzymes; Schnee et al., 2006; Tholl, 2006). Hence, multivariate analyses are more appropriate to extract the biologically relevant information from VOC blends than multiple single ANOVAs, which ignore these internal correlations. Last but not least, multivariate analyses provide a better understanding of the system because they summarize the variation of potentially hundreds of compounds in a limited number of - typically two or threefactors. These consist of 'scores' that are indicative for the compositional difference of VOC blends for each subject (plant), while the relative importance of each VOC in a factor is quantified by model 'loadings' (Jansen et al., 2010). Scores and loadings can be plotted in twodimensional figures that provide attractive visual support for whether and how different VOC profiles differ from each other.

Two types of multivariate models can be distinguished based on their objective: unsupervised models, of which Principal Component Analysis (PCA) is most widely used, describe all information in the data as well as possible. Different origins of the information (e.g., experimentally induced or stochastic variation) are not distinguished. Supervised methods, on the other hand, focus on a priori defined differences between plants, corresponding to treatments imposed by the experiment. Supervised models therefore are more appropriate to distinguish differences between VOC blends emitted by plants that are experimentally induced (Jansen et al., 2010). Partial Least SquaresDiscriminant Analysis (PLSDA) is the method that is most widely used to this end in metabolomic analyses (Barker and Rayens, 2003). This model consists of a 'prediction' of whether each plant was treated or not, and quantifies the importance of each VOC in the separation between treatment groups. This latter quantification is referred to as a Discriminant Function. PLSDA is often applied to dichotomous problems, for example, to compare induced with control plants. However, choice tests in biological experiments often are more complex than that and require 
multiple comparisons between treatment groups. This can be solved by defining 'protected contrasts' between more than two groups based on experimentally or biologically relevant combinations of the treatments, as is often employed in analysis of variance models (Sokal and Rohlf, 1995). We employed such protected contrasts in our PLSDA model to explicitly correlate consistent shifts in the VOC profiles due to root or shoot induction with the host plant choices of parasitic wasps.

Here, we present a case study to illustrate how the novel combination of PLSDA with protected contrasts can identify biological relevant shifts in complex VOC blends. The aim of the analysis is: 1) to identify shifts in VOC blends elicited by shoot feeding herbivores on feral cabbages (Brassica oleracea) that were previously induced at the roots or the shoots; and 2) to link these shifts to parasitoid preference data in a statistically sound and visually attractive fashion. For the initial induction of the cabbage plants, we used jasmonic acid (JA), an endogenous plant hormone that is part of the signaling cascade induced by chewing insects and biotrophic leaf pathogens (De Vos et al., 2005). External JA application triggers various herbivore-induced responses in Brassica spp. including VOC emissions that attract natural enemies to the plant (Loivamäki et al., 2004). Here, we induced the cabbage plants either via the roots or on the shoots. The use of JA as the initial inducer has the advantage that both roots and shoots can be induced quantitatively and in a similar fashion, which is not achievable when using real insect herbivores. For our case study, this experimental advantage was prioritized over the fact that external JA application does not exactly mimic herbivore induced VOC in cabbages (Bruinsma et al., 2009). Three days after JA treatment, we placed ten Pieris brassicae or ten P. rapae caterpillars on the leaves of each plant to analyze the effects on JA pretreatment on aboveground VOCs induced by natural herbivore damage. Both caterpillar species are known to induce the production of similar groups of VOCs in various cabbage species, but differ in the amount of damage they do (Geervliet et al., 1997; Smid et al., 2002). Plants with caterpillars were presented in pairs or triplets to female Cotesia glomerata, a gregarious parasitoid wasp that frequently parasitizes both Pieris species in the field (Brodeur and Vet, 1995; Geervliet et al., 2000). Similarly treated plants with caterpillars were used to collect the dynamic headspace of control, root JA-induced and shoot JA-induced plants on direct thermodesorption traps. After GC-MS analyses of the VOC profiles, we used the experimental design plus the hierarchical preferences of the parasitoids to develop a PLSDA model combined with contrast analyses to reveal which shifts in the VOC blends may explain the wasps' behavior.

\section{Methods and Materials}

Plant Growth and Induction Feral Brassica oleracea L. (Brassicacea) seeds from a batch that was originally collected from several individuals in a wild population near Heteren (see van Dam et al., 2004) and propagated for one generation in the communal garden at the NIOO (open pollination), were germinated on glass beads. After 1 week, the seedlings were transferred to 1.31 pots filled with a sand-peat mixture. Plants were watered with tap water as needed. When plants had six true leaves, they were assigned to the following treatment groups: shoot jasmonic acid induction (SJA); root JA induction (RJA); or control (CON). Plants in the SJA group received $500 \mu \mathrm{g}$ jasmonic acid (JA; Sigma, St Louis, IL, USA) in a $0.1 \%$ Triton X100 solution on the oldest two leaves, and the RJA group received $500 \mu \mathrm{g} \mathrm{JA}$ in $10 \mathrm{ml} 0.1 \%$ Triton solution injected in the soil near the roots. CON plants and untreated organs of the JA-induced plants were treated with similar volumes of acidic solution ( $\mathrm{pH}=3.7$ with $\mathrm{HCl} ; 0.1 \%$ Triton) to control for acid induced responses (see van Dam et al., 2004 for a detailed description of the procedure). This amount of JA has been shown to increase leaf glucosinolates 3-7 day after application (Qiu et al., 2009). The control plants received a solution with a similar $\mathrm{pH}$ to control for induction effects due to the acidity of the JA solution.

Parasitoid Choice Tests Pieris brassicae L. (Lepidoptera; Pieridae; large white) and P. rapae L. (Lepidoptera; Pieridae; small white) caterpillars were obtained from a culture maintained on B. oleracea cv. gemmifera var. Cyrus at the Laboratory of Entomology, Wageningen University, The Netherlands. Three days after the JA treatments, 10 first instar larvae per plant were placed on two untreated leaves. The larvae were allowed to feed for at least $3 \mathrm{~h}$ before plants were used in the choice experiment. Cotesia glomerata (Hymenoptera; Braconidae) wasps were obtained from parasitized $P$. brassicae larvae reared as above. After emergence from the cocoons, adult wasps were kept in a communal cage with access to honey and water. After 3-6 day, female wasps were isolated from the culture and given a single oviposition experience with a first instar caterpillar presented on a paint brush (Smid et al., 2007). The experienced wasps then were introduced singly via a sliding observation window into a cage $(100 \times$ $70 \times 70 \mathrm{~cm}$ ) covered with fine mesh gauze on three sides and the roof. Two plants with caterpillars were placed $30-40 \mathrm{~cm}$ apart in the back of the cage. When there were three plants, they were placed in a triangular configuration $(30 \mathrm{~cm}$ apart). Wasps were released individually on the bottom of the cage equidistant to all plants. Per set of plants, 10-15 female wasps were tested for their preference. After five tests, the 
positions of the plants were swapped. Individual plants were used for one set only. The preference observations were performed over several days. On each day, at least one replicate of each possible treatment combination was included to control for day-effects. A positive choice was scored as the first landing of the wasp on a plant, after which the wasp was removed immediately. Individual wasps were used only once for a preference test. The preference data were analyzed with replicated G-tests (Sokal and Rohlf, 1995). Within each comparison, each set of plants and the 10-15 females that chose between them, were considered as one replicate in the G-test.

Volatile Trapping and GC-MS Analysis A separate group of plants was treated as above with JA. Three days later, CON, SJA, and RJA groups all received 10 caterpillars on two untreated leaves. The plants were transferred individually to 171 glass bell jars $(41 \mathrm{~cm}$ high, $24.5 \mathrm{~cm}$ diam) placed on a stainless steel bottom plate in a controlled climate cabinet $\left(21^{\circ} \mathrm{C}, 70 \%\right.$ r.h. $)$. At the top, the jars were supplied constantly with pressurized air $(200 \mathrm{ml} / \mathrm{min}$; Hoekloos, Rotterdam, The Netherlands) cleaned over a Zero Air generator to remove hydrocarbons (Parker Hannifin Corp, Tewksbury, MA, USA). Volatiles were collected by pulling headspace air over a steel trap filled with $150 \mathrm{mg}$ Tenax TA and $150 \mathrm{mg}$ Carbopack B (Markes International Ltd., Llantrisant, UK) with a vacuum pump. Traps were mounted at shoot level to an outlet on the side of the jar. Flow rates over the traps were set to $100 \mathrm{ml} / \mathrm{min}$ using mass flow regulators (Sho rateTM, Brooks Instrument, Hatfield, PA, USA). After 45-60 min, traps were removed, capped, and stored at $4^{\circ} \mathrm{C}$ till analysis. Four jars with individual mass flow regulators were sampled in parallel. Six to seven full series of 4 plants and two background VOC profiles from an empty jar were sampled between 9:00 and 17:00 $\mathrm{h}$. Thus, we sampled the following numbers of individual plants in each experiment: P. brassicae experiment $15 \mathrm{CON}$, 14 SJA, 15 RJA, and 6 untreated plants without caterpillars for background trapping; P. rapae experiment: $13 \mathrm{CON}, 13$ SJA, 13 RJA, and 12 untreated plants without caterpillars.

VOCs were desorbed from the traps using an automated thermodesorption unit (model Unity, Markes International Ltd., Llantrisant, UK) at $200^{\circ} \mathrm{C}$ for $12 \mathrm{~min}$ (He flow $30 \mathrm{ml} /$ min) and focused on an internal Tenax cold trap $\left(-10^{\circ} \mathrm{C}\right)$. After 1 min of dry purging, trapped VOCs were introduced into the GC-MS (model Trace, ThermoFinnigan, Austin, TX, USA) by heating the cold trap for $3 \mathrm{~min}$ to $270^{\circ} \mathrm{C}$. Split ratio was set to $1: 4$, and the column used was a $30 \mathrm{~m} \times$ $0.32 \mathrm{~mm}$ ID RTX-5 Silms, film thickness $0.33 \mu \mathrm{m}$ (Restek, Bellefonte, PA, USA). Temperature program: from $40^{\circ} \mathrm{C}$ to $95^{\circ} \mathrm{C}$ at $3{ }^{\circ} \mathrm{C} / \mathrm{min}$, then to $165^{\circ} \mathrm{C}$ at $2^{\circ} \mathrm{C} / \mathrm{min}$, and finally to $250^{\circ} \mathrm{C}$ at $15^{\circ} \mathrm{C} / \mathrm{min}$. The VOCs were detected by the MS operating at $70 \mathrm{eV}$ in EI mode. Mass spectra were acquired in full scan mode (33-300 AMU, $0.4 \mathrm{scan} / \mathrm{sec})$. Compounds were identified by their mass spectra using deconvolution software (AMDIS) in combination with NIST 2005 (National Institute of Standards and Technology, USA, http://www.nist.gov) and Wiley 7th edition spectral libraries. Additionally, mass spectra (minimum match factor $=85 / 100$ ) and linear retention indices (LRI, window set to $+/-2$ ) of chromatographic peaks were compared with values reported in the literature (Adams, 2007), based on which we compiled a database of over 13.000 literature LRI entries from studies using similar phases as the RTX-5 column (5\% phenyl equivalent polysilphenylene-siloxane). Additionally, we obtained reference spectra from several authentic standards (farnesene, limonene, methyljasmonate, methylsalicylate, dimethyldisulfide, dimethyltrisulfide, octanal, nonanal, decanal, cis-3hexen-1-ol, 2-phenylethylalcohol, indole, benzylcyanide, and phenylethylisothiocyanate; Sigma- Aldrich, St. Louis, Il, USA). The integrated signals generated by the AMDIS software from the MS-chromatograms were used for comparison among the treatments. Peak areas in each sample were divided by the total volume in $\mathrm{ml}$ that was sampled over the trap to correct for differences in sampling volume between experiments.

Multivariate Data Analysis of VOC Data Only those VOCs with a peak area larger than the background and found in more than $50 \%$ of the samples of either treatment group were selected for multivariate analysis. Thus, in the P. brassicae experiment 116 compounds, and in the P. rapae experiment 136 compounds were statistically analyzed. Prior to analysis, the P. rapae data were transformed as $\log (x+1)$ and for both caterpillar spp. the data were unitvariance scaled. We analyzed two contrasts that were of interest: the difference between all JA-treated and control plants (CON vs. (SJA+RJA)), and between RJA and SJA plants. These two 'protected contrasts' were modeled using two separate Partial Least Squares Discriminant Analysis (PLSDA) models per caterpillar species (Barker and Rayens, 2003). The first contrast describes the general effect of JA application, regardless of where the JA was applied, by contrasting the VOC profiles emitted by plants in the control group against those emitted by both JAtreated groups (CON vs. (RJA-SJA), lower horizontal axes in Fig. 2a, b). This axis thus indicates which VOCs explain the preference of parasitic wasps for JA-induced plants in general. The second contrast was that between the SJA and RJA plants, thus revealing which VOCs are responsible for the parasitoids' preference for shoot induced plants. For validation purposes, 1000 models with randomly permuted class labels were fitted alongside every model. The number of latent variables required for both models was determined using cross-validation (Geladi and Kowalski, 1986). To rule 
Fig. 1 Preference of Cotesia glomerata wasps expressed as the percentage of wasps preferring the plant $( \pm$ s.e.m. calculated over 8-18 replicate test runs of $10-15$ females per run). $\mathbf{a}$ and c Dual-choice tests between plants treated with jasmonic acid (JA) to the roots (RJA), to the shoots (SJA) or with acidic water $(\mathrm{CON}) 3 \mathrm{~d}$ before 10 Pieris brassicae (a) or 10 Pieris rapae (c) larvae were added. Asterisks denote a significant difference in preference between the two plants $(* \mathrm{P} \mathrm{Gp}<0.01$, ** P Gp $<0.001$, see Table 1. b and d) Triple choice tests between CON, RJA and SJA plants. Asterisks denote an overall significance in preference between the three plants (** $\mathrm{P} \mathrm{Gp}<0.001)$
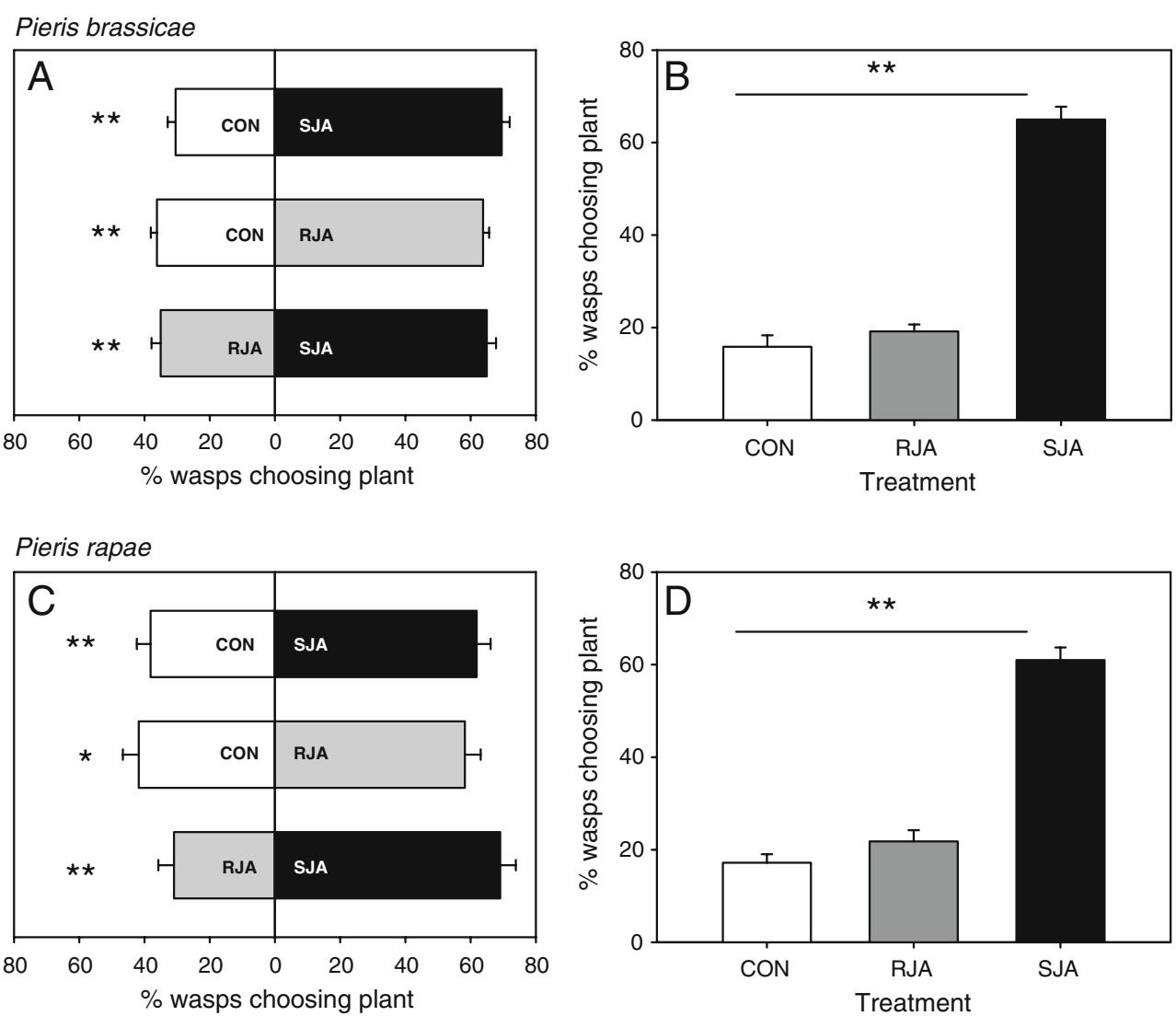

out model over-fit, the predictive capacity of each model from the cross-validation was compared to that of permuted models of equal complexity. The permutation test also was used to determine the likelihood of each VOC being involved in the modeled contrasts: this is done by comparing the value of the discriminant function for each VOC to those of the same VOC in the discriminant functions of the randomly permuted data sets (which form a 'null distribution'). This comparison can be translated into a $P$-value for each VOC in each contrast.

\section{Results}

In dual choice tests, 5-day old Cotesia glomerata females always preferred JA-induced plants over control plants, regardless of where the JA was applied or the Pieris species that was used (Fig. 1a, c; Table 1). However, when given the choice, wasps consistently and significantly preferred SJA plants over RJA plants, irrespective of the caterpillar species that was feeding on the leaves (Fig. 1a,c). This strong and significant preference for SJA plants also was evident when plants from the three different treatment groups were presented simultaneously to the wasps (Fig. 1b, d).
In both VOC analyses, the first discriminant axis represents the contrast between the control and both the RJA and SJA plants (Fig. 2a, b, horizontal axes). The importance of each VOC in this contrast is represented by the horizontal position of the compound, and its significance for each contrast is indicated by both point labels. This showed that the increased emission of monoterpenes is shared by RJA and SJA plants (compounds 6-15 in Fig. 2a, $\mathrm{b}$, and Table 2), which contributed highly to the separation of both JA-treated groups from the CON group. The induction of monoterpene emissions by JA-application was consistent for both Pieris species (Fig. 3). One VOC decreased after JA application: in the P. brassicae experiment, the emissions of dimethyldisulfide (DMDS) were lower in JA-treated plants (compound 5 in Fig. 2a). Significant decreases in DMDS emissions in JA-induced plants were not observed in the $P$. rapae experiment.

Interestingly, we found a clear contrast in VOC profiles between root and shoot JA-induced plants (RJA vs. SJA; vertical axes in Fig. 2). Shoot JA application strongly induced the emission of several sesquiterpenes (compounds number 16-18, 20-23) and a homoterpene (number 19), whereas root induction did not result in elevated emissions of these compounds (Table 2; Fig. 2a, B; Fig. 4a, b). Again, this pattern was similar for both herbivore experiments and was also observed for unidentified sesquiterpenes (Table 2, 
Table 1 Goodness-of-fit tests (g-test) of Cotesia glomerata preference

\begin{tabular}{lclll}
\hline Test combination & Replicates $^{\mathrm{a}}$ & Total wasps $^{\mathrm{b}}$ & $\mathrm{P} \mathrm{Gh}^{\mathrm{c}}$ & $\mathrm{P} \mathrm{Gp}^{\mathrm{d}}$ \\
\hline Caterpillar : Pieris & brassicae & & & \\
CON_SJA & 13 & 191 & 0.91 & $<\mathbf{0 . 0 0 1}$ \\
CON_RJA & 13 & 196 & 0.99 & $<\mathbf{0 . 0 0 1}$ \\
RJA-SJA & 13 & 198 & 0.72 & $\mathbf{0 . 0 0 1}$ \\
CON_RJA- & 13 & 197 & 0.84 & $<\mathbf{0 . 0 0 1}$ \\
SJA & & & & \\
Caterpillar: Pieris & rapae & & & \\
CON-SJA & 16 & 180 & 0.09 & $<\mathbf{0 . 0 0 1}$ \\
CON-RJA & 15 & 171 & 0.07 & $\mathbf{0 . 0 1 7}$ \\
RJA-SJA & 18 & 140 & $\mathbf{0 . 0 0 3}$ & $<\mathbf{0 . 0 0 1}$ \\
CON-RJA-SJA & 8 & 120 & 0.98 & $<\mathbf{0 . 0 0 1}$ \\
\end{tabular}

a. Number of replicates (plant pairs). b. numbers of wasps tested. c. P $\mathrm{Gh}$ indicates whether the replicates are heterogeneous; if $\mathrm{P} \mathrm{Gh}>0.05$ the choice of the different sets of wasps has been consistent over the replicates. d. P Gp indicates that, based on the pooled results the nullhypothesis stating that wasps have no preference for one of the treatments may be rejected. $\mathrm{P}$-values $<0.05$ are in bold

CON control

$S J A$ shoot jasmonic acid induction

$R J A$ root jasmonic acid induction

Fig. 4b). The levels of a few compounds were lower after shoot than after root JA treatment; in the P. brassicae experiment, several aldehydes (compounds 1-3), and in the

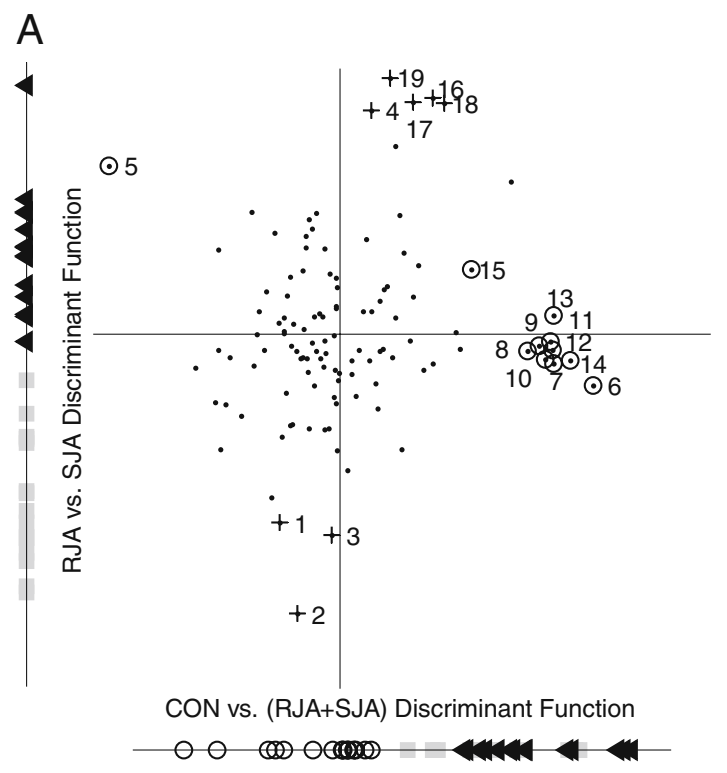

Fig. 2 Two-dimensional PLS-Discriminant fingerprints for VOCs emitted by Brassica oleracea plants with a. 10 Pieris brassicae larvae or b 10 P. rapae larvae. The separation of individual plants on each discriminant function are given on the left vertical (RJA vs. SJA) and lower horizontal (CON vs. (RJA+SJA)) axes in each figure. Open circles $=$ control plants, grey squares $=$ root-induced $($ RJA) plants; black riangles $=$ shoot-induced (SJA) plants. The two-dimensional plots in the middle show the contribution of each VOC to the separation. The
P. rapae experiment, 2,4-pentadione (compound 24), were more pronounced in control and root induced than in shoot induced plants (Table 2, Fig. 2a, b). In contrast to the differences in terpene emissions, these effects were observed for only one of the Pieris species.

\section{Discussion}

Based on the results of our combined PLSDA-protected contrast analysis, we could easily identify which VOCs are associated with parasitoid preference. In all choice experiments, C. glomerata females strongly preferred aboveground JA-induced plants that had the highest emissions of sesqui- and homoterpenes, even when neighboring belowground induced plants showed equally increased monoterpene levels. This indicates that sesquiterpenes are more attractive cues for this parasitoid species than monoterpenes are. This does not preclude that monoterpenes themselves are attractive as well, but our results show that their attractiveness was context-dependent. The emission of various VOCs from other biosynthetic classes changed after JA application as well, but only the shifts in terpene emissions were consistent for both experiments. Several other studies have shown that plants with enhanced levels of monoterpenes or sesquiterpenes/homoterpenes are more attractive to C. glomerata females (van Poecke et al., 2001;

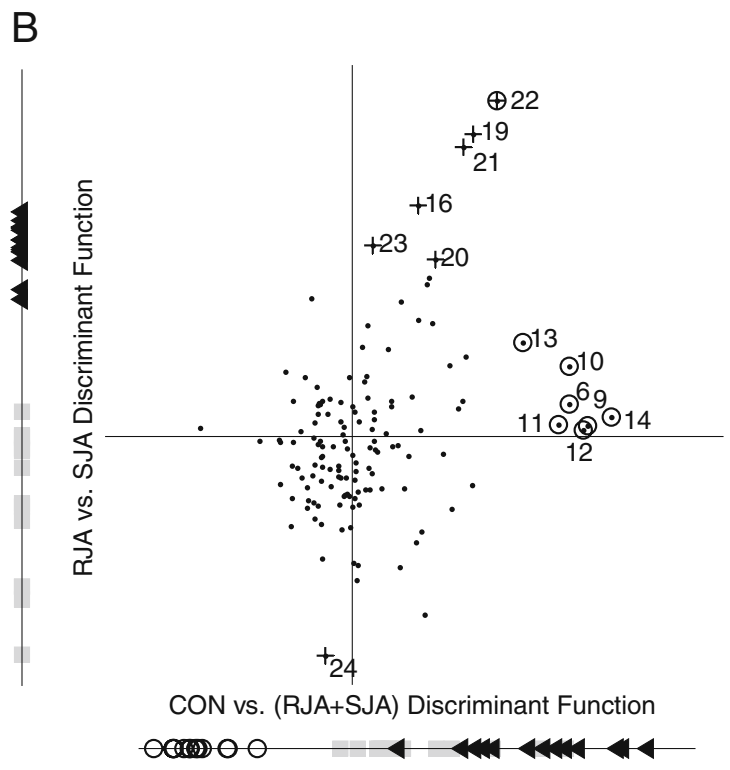

position of each point is determined by its importance for the contrasts. The numbered VOCs (see Table 1) contributed significantly to the separation of treatment groups (encircled: (CON vs. (RJA+ SJA)), crosses: for (RJA vs. SJA). Statistical significance was determined by testing the discriminant function value of a VOC against a null distribution of 1000 models on randomly permuted treatment group assignments $(P<0.05)$ 
Table 2 Differentially induced volatile organic compounds in the headspace of jasmonic acid induced feral Brassica oleracea plants

\begin{tabular}{|c|c|c|c|c|c|c|c|c|}
\hline \multirow[b]{2}{*}{ No. } & \multirow[b]{2}{*}{ Compound name ${ }^{a}$} & \multirow[b]{2}{*}{$\mathrm{RI}^{\mathrm{b}}$} & \multirow[b]{2}{*}{ Reported in ${ }^{\mathrm{c}}$} & \multirow[b]{2}{*}{ Compound class } & \multicolumn{2}{|c|}{ Pieris brassicae } & \multicolumn{2}{|c|}{ Pieris rapae } \\
\hline & & & & & $\mathrm{P}(\mathrm{CON}-\mathrm{JA})^{\mathrm{d}}$ & P(RJA-SJA) & $\mathrm{P}(\mathrm{CON}-\mathrm{JA})$ & P(RJA-SJA) \\
\hline 1 & Nonanal* & 1103 & A, B & aldehyde & 0.2698 & 0.0400 & - & - \\
\hline 2 & Decanal* & 1204 & $\mathrm{~A}, \mathrm{~B}$ & aldehyde & 0.3606 & 0.0308 & - & - \\
\hline 3 & 2-Undecanal & 1362 & $\mathrm{~A}, \mathrm{~B}$ & aldehyde & 0.4718 & 0.0388 & - & - \\
\hline 4 & Hexylacetate & 1016 & $\mathrm{~A}, \mathrm{~B}$ & ester & 0.3520 & 0.0110 & - & - \\
\hline 5 & Dimethyldisulfide* & 738 & $\mathrm{~A}, \mathrm{~B}$ & sulfide & 0.0330 & 0.0890 & - & - \\
\hline 6 & 1,8-Cineole & 1028 & $\mathrm{~A}, \mathrm{~B}$ & monoterpene & 0.0024 & 0.3500 & 0.0098 & 0.2982 \\
\hline 7 & alpha-Pinene & 927 & $\mathrm{~A}, \mathrm{~B}$ & monoterpene & $<0.001$ & 0.3238 & - & - \\
\hline 8 & alpha-Terpinolene & 1084 & $\mathrm{~A}, \mathrm{~B}$ & monoterpene & 0.0172 & 0.4698 & - & - \\
\hline 9 & alpha-Thujene & 921 & $\mathrm{~A}, \mathrm{~B}$ & monoterpene & $<0.001$ & 0.4254 & 0.0056 & 0.4456 \\
\hline 10 & beta-Myrcene & 990 & A, B & monoterpene & $<0.001$ & 0.3354 & 0.0228 & 0.2496 \\
\hline 11 & Limonene* & 1027 & $\mathrm{~A}, \mathrm{~B}$ & monoterpene & $<0.001$ & 0.4564 & 0.0156 & 0.4218 \\
\hline 12 & Sabinene & 967 & A, B & monoterpene & $<0.001$ & 0.3988 & 0.0088 & 0.4612 \\
\hline 13 & (E)-Sabinene hydrate & 1094 & $\mathrm{~A}, \mathrm{~B}$ & monoterpene & 0.0156 & 0.4602 & 0.0292 & 0.1934 \\
\hline 14 & (Z)-Sabinene hydrate & 1064 & & monoterpene & 0.0012 & 0.3634 & 0.0052 & 0.3968 \\
\hline 15 & Monoterpene 1 & 1024 & & monoterpene & 0.0418 & 0.2138 & - & - \\
\hline 16 & beta-Elemene & 1389 & A, B & sesquiterpene & 0.1300 & $<0.001$ & 0.2832 & 0.0074 \\
\hline 17 & Zingiberene & 1491 & & sesquiterpene & 0.2270 & 0.0068 & - & - \\
\hline 18 & Sesquiterpene 1 & 1500 & & sesquiterpene & 0.1222 & 0.0024 & - & - \\
\hline 19 & $\begin{array}{l}\text { (3E)-4,8-Dimethyl-1,3, } \\
\text { 7-nonatriene (DMNT) }\end{array}$ & 1117 & B & homoterpene & 0.2926 & 0.004 & 0.1232 & 0.0024 \\
\hline 20 & Sesquiterpene 2 & 1460 & & sesquiterpene & - & - & 0.2076 & 0.0216 \\
\hline 21 & Sesquiterpene 3 & 1464 & & sesquiterpene & - & - & 0.1120 & $<0.001$ \\
\hline 22 & Sesquiterpene 4 & 1467 & & sesquiterpene & - & - & 0.0458 & $<0.001$ \\
\hline 23 & Sesquiterpene 5 & 1469 & & sesquiterpene & - & - & 0.4204 & 0.0118 \\
\hline 24 & 2,4-Pentadione & 779 & & ketone & & & 0.4082 & 0.0374 \\
\hline
\end{tabular}

a. The table contains only those volatile organic compounds (VOCs) that were found to significantly contribute to the separation of the treatment groups. The numbers in the first column refer to the numbers in the contrast plot in Fig. 2a and b. Compounds indicated with a $*$ were identified by comparison of pure standards, the other compounds were identified based on MS reference manuals (Adams, 2007), NIST 2005, Wiley 7th Ed. MS library, and our own MS and LRI literature library. All plants had 10 Pieris brassicae or 10 P. rapae caterpillars feeding on their shoots at the time of VOC collection. b. RI=retention index on the GC-MS; column RTX5-MS. One monoterpene and five sesquiterpenes could not be sufficiently identified based on their RI value and mass spectrum using our criteria, and were given sequential numbers. c. indicates whether this compound had been reported earlier in various Brassica species (B, based on references Bruinsma et al., 2009; Geervliet et al., 1997; Gols et al., 2008; Soler et al., 2007) or Arabidopsis (A, based on reference Rohloff and Bones, 2005). d.The P-values were determined by testing the discriminant function value of a VOC against a null distribution of 1000 models on randomly permuted treatment group assignments. Values in bold are $P<0.05$

Mumm et al., 2008). The strength of our approach, however, lies in the fact that we could readily contrast the relative importance of these two terpene classes within the VOC blend and could directly relate differences in VOCs between JA-treatments to parasitoid choice behavior. Our analysis, however, cannot reveal whether one, several, or all of the terpenes together are responsible for the observed preferences. This could be assessed experimentally by presenting isolated compounds - in combination with the appropriate background odors - to parasitoids in choice tests, or by using plants that are genetically modified with terpene synthase genes (Kessler and Baldwin, 2001; Ibrahim et al., 2005; Kappers et al., 2005; Mumm and
Hilker, 2005). The latter approach has shown that single terpene synthases generally yield mixtures of similar terpenes within a class (Kappers et al., 2005; Schnee et al., 2006; Tholl, 2006). This is consistent with the induction patterns we saw in our experiments, in which several members of a biosynthetic class responded uniformly to the induction treatments. The absence of a strong selection on terpene synthases to produce a single product, underscores our assumption that plant $\mathrm{VOC}$ cues likely consist of 'bouquets' of different VOCs, and that it is the quality of the blend rather than the quantity of a single compound that determines the behavioral response (van Poecke et al., 2001). 

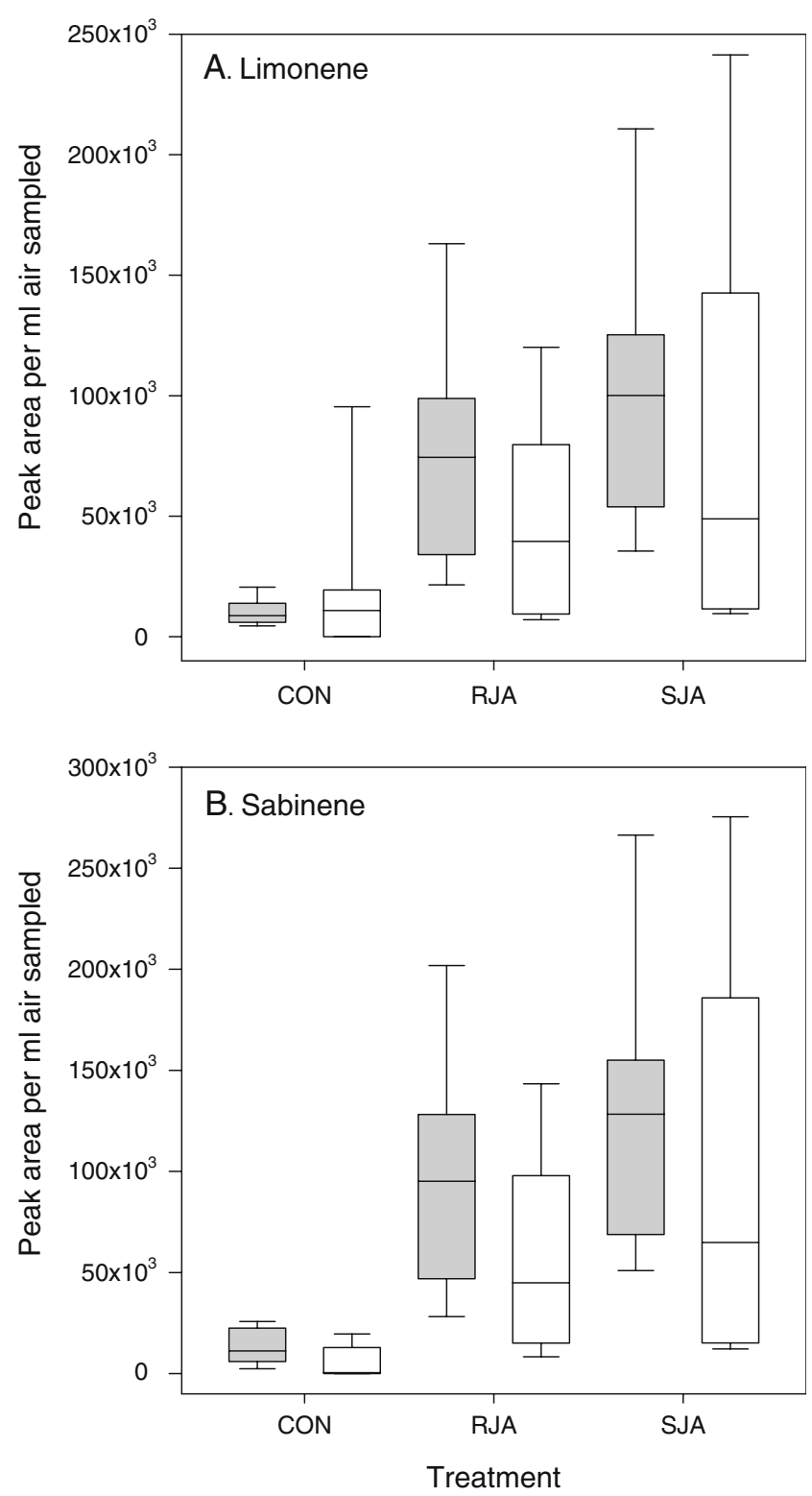

Fig. 3 Box plots showing the median value, the 25th percentile, and 75th percentile of the peak area per ml sampled air for a limonene, and b sabinene. Grey bars: values in Pieris brassicae experiment, white bars: values in $\mathrm{P}$. rapae experiment. $\mathrm{CON}=$ control plants, $\mathrm{RJA}=$ root jasmonic acid (JA) induced plants, SJA=shoot JA induced plants. The error bars above and below the box indicate the 90th and 10th percentile

As expected, JA application only partly mimicked herbivore-induced responses. Similar to the induced responses caused by root herbivore damage, only shoot induction with JA significantly increased the emission of sesqui- and homoterpenes, whereas root herbivory and root JA application did not (Soler et al., 2007). JA application to the roots, however, did not increase the emissions of sulfides, such as DMDS, which have been found to be induced by cabbage root fly larvae. These sulfides also are of biological relevance, but they play a dual role. It has been suggested that $C$. glomareta wasps use these as cues to avoid root infested plants (Soler et al., 2005), whereas soil dwelling beetle predators of root fly larvae, and parasitoids of Plutella xylostella, another leaf feeding cabbage specialist, may use DMDS as a positive cue to locate their host or prey (Ferry et al., 2007; Kugimiya et al., 2010). As JA application did not increase the emission of sulfide compounds in root induced plants, we could not asses the ecological role of this compound in our set-up. For $C$.
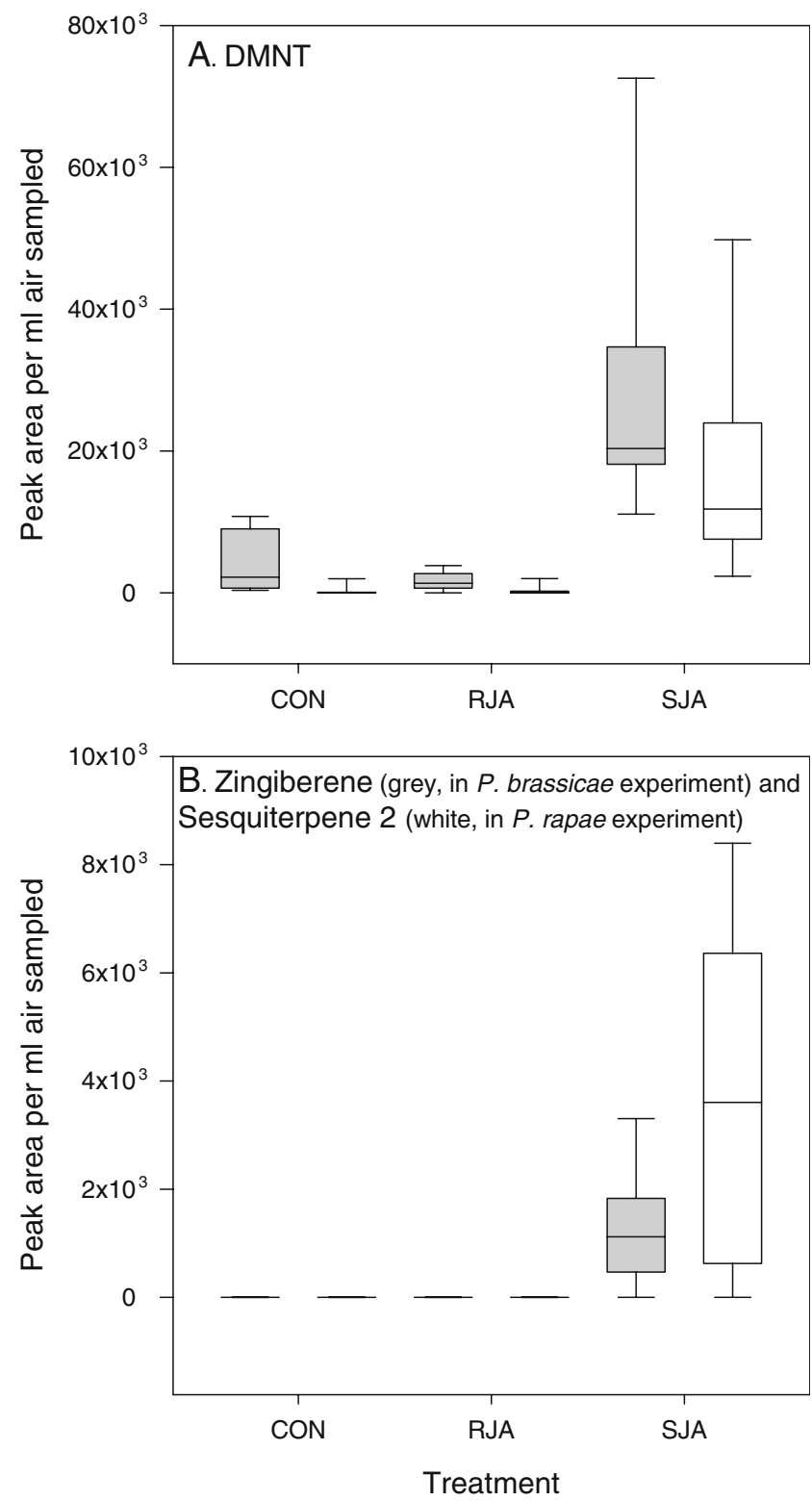

Fig. 4 Box plots showing the median value, the 25th percentile, and 75th percentile of the peak area per ml sampled air for homo-and sesquiterpenes. a DMNT.' $<3 \mathrm{E}>-4,8$-dimethyl-1,3,7-nonatriene; b Zingiberene plus sesquiterpene 2 (zingiberene alike); Legend (DMNT) Grey bars: values in Pieris brassicae experiment, white bars: values in P. rapae experiment. $\mathrm{CON}=$ control plants, $\mathrm{RJA}=$ root jasmonic acid (JA)-induced plants, SJA=shoot JA-induced plants. The error bars above and below the box indicate the 90th and 10th percentile 
glomerata, it is of ecological and evolutionary relevance to assess the difference between root and shoot-infested plants, as root-infested plants yield hosts of inferior quality or her offspring. Wasps emerging from caterpillars on rootinfested plants attain lower body masses, which eventually results in a lower fitness (Soler et al., 2005). In an earlier study, we found that parasitoid fitness parameters were reduced significantly on shoot JA-induced plants (Qiu et al., 2009). Yet, we found that shoot JA-induced plants were strongly preferred by female wasps. The discrepancy between parasitoid performance and preference may be explained partly by the fact that JA-induction does not provide the full complement of volatile cues that this parasitoid needs to locate the most suitable hosts.

There is ample evidence that female $C$. glomerata wasps have the ability to detect terpenes and various plant odors with their antennae and can learn to prefer different odors when rewarded (Vet et al., 1995; Smid et al., 2002, 2007). Much less is known about how parasitoids perceive and integrate compositional shifts in complex VOC mixtures, and how they learn to respond to these changes within complex odor plumes (but see Vet and Groenewold, 1990). Learning more about sources of variation in plant odor signals is important for our understanding of the evolution of plant-parasitoid interactions and the role of VOC therein (Vet, 1999; Allison and Hare, 2009; Schuman et al., 2009). The signal-to-noise ratio of the odor profiles determines the reliability of the information, and this may influence the way parasitoids respond to plant cues, innately or through learning. Vice versa, the way parasitoids perceive and learn to distinguish between plant VOCs may influence the evolution of plant VOC emissions with regards to the quantitative and qualitative composition of the odor blend in response to herbivore damage (Vet, 1999; Allison and Hare, 2009). Our study demonstrates that parasitoids are able to specifically detect enhanced emissions of sesquiand homoterpenes against a background of equally increased monoterpene emissions in JA-induced plants. This suggests that the presence of especially sesqui- and homoterpenes in the VOC mixtures may serve as a reliable cue that signals the presence of shoot induction by actively feeding herbivores on a plant that is free from root herbivores.

Unexpectedly, our results also reveal that the terpene synthases of the cytosolic mevalonate (MVA) pathway, leading to the synthesis of sesqui- and homoterpenes (Degenhardt and Gershenzon, 2000; Hopke et al., 1994; Tholl, 2006), are induced by shoot JA application only. In contrast, the biosynthesis of monoterpenes via the methylerythritol phosphate (MEP) pathway, which is located in the plastid, is equally elicited by root and shoot JA application. It has been known longer that terpene and sesquiterpene synthases are induced by internal as well as external JA
(Ozawa et al., 2000; Tholl, 2006). However, it was hitherto unknown that terpene synthases belonging to these two major biosynthetic pathways may respond differently to JA application, solely depending on where the JA was applied on the plant. It is unlikely that the differential induction of mono- and sesquiterpenes is merely a matter of signal dilution in root induced plants. First, monoterpene emissions were equally enhanced in root and shoot JA-induced plants. Moreover, similar regulatory differences have been found for the induction of glucosinolates, which serve as direct defense compounds in Brassicaceae (Hopkins et al., 2009). Shoot JA induction increased the levels of indole glucosinolates that are derived from tryptophan, whereas root induction triggered the production of the aliphatic glucosinolates (van Dam et al., 2004; Jansen et al., 2009). The latter are produced from methionine via a different biosynthetic and regulatory pathway. Taken together, these results imply that root induced responses are regulated fundamentally different from responses triggered by shoot herbivory. This may provide plants with the option to optimize their inducible defense responses in natural environments where both root and shoot herbivores may interact with the plant (Bezemer and van Dam, 2005). Eventually, the differential expression of both direct and indirect induced defenses after root and shoot herbivory may have profound effects on the performance of aboveground herbivores and parasitoids associated with plants (Masters et al., 2001; Soler et al., 2005; Qiu et al., 2009). In addition to population-based variation in the sensitivity to induction hormones (Schuman et al., 2009), root induction may be yet another level of variation that determines differences in induced VOC profiles between plants.

Here, we have shown how novel bioinformatic analyses successfully bridge the gap between ecologically relevant observations and JA-induced changes in VOC blends. Our supervised PLSDA coupled to protected contrast analysis provided strong statistical support as well as visually appealing information on shifts in chemically complex VOC profiles. It enabled us to assess the relative importance of shifts in different VOC blends and directly link these to the behavior of higher trophic levels associated with these plants. This could not have been achieved with a conventional discriminant analysis that focused only on how each treatment group differed from all other groups. We expect that this method can be applied easily to similar experiments with real herbivores, which may yield novel information regarding the relative importance of specific (groups) of VOCs that are differentially induced by aboveground and belowground induction. The results also show that multivariate analyses do not only provide answers to experimental questions, but also may provide additional insight and generate novel, testable hypotheses about the internal regulation and coordination of above- 
ground and belowground induced responses in plants. Bioinformatic analyses, thus, are an invaluable tool to 'see the woods from the trees' that should be applied more frequently to analyze plant VOC blends and other complex chemical data sets.

Acknowledgements The authors thank C.E. Raaijmakers and G. Disveld at NIOO-KNAW for assistance, L. Koopman, A. Gidding of the laboratory of Entomology Wageningen University for providing Pieris eggs, and S. Schaper, NIOO-KNAW for setting up the first sets of parasitoid observations. J. Harvey, NIOO-KNAW, provided valuable comments on an earlier version of this manuscript. NM van Dam thanks the Netherlands Organization for Scientific Research (NWO) for funding her work with a VIDI grant, no. 864-02-001, and B-L Qiu was granted by the China Scholarship Programs no. [2006]3036.

Open Access This article is distributed under the terms of the Creative Commons Attribution Noncommercial License which permits any noncommercial use, distribution, and reproduction in any medium, provided the original author(s) and source are credited.

\section{References}

ADAMS, R. P. 2007. Indentification of essential oil components by gas chromatography/mass spectrometry. Carol Stream, IL, USA: Allured Publishing Corporation.

Allison, J. D., and HARE, J. D. 2009. Learned and naive natural enemy responses and the interpretation of volatile organic compounds as cues or signals. New. Phytol. 184:768-782.

BARGMANN, C. I. 2006. Comparative chemosensation from receptors to ecology. Nature 444:295-301.

BARKER, M., and RAYENS, W. 2003. Partial least squares for discrimination. J. Chemometr. 17:166-173.

BEZEMER, T. M., and VAN DAM, N. M. 2005. Above- belowground interactions via induced plant defenses. Trends Ecol. Evol. $20: 617-624$.

Brodeur, J., and Vet, L. E. M. 1995. Relationships between parasitoid host range and host defence: a comparative study of egg encapsulation in two related parasitoid species. Physiol. Entomol. 20:7-12.

Bruinsma, M., Posthumus, M. A., Mumm, R., Mueller, M. J., VAN LOON, J. J. A., and DiCKE, M. 2009. Jasmonic acid-induced volatiles of Brassica oleracea attract parasitoids: effects of time and dose, and comparison with induction by herbivores. J. Exp. Bot. 60:2575-2587.

De Vos, M., Van Oosten, V. R., Van Poecke, R. M. P., Van Pelt, J. A., Pozo, M. J., Mueller, M. J., Buchala, A. J., MetrauX, J. P., VAN LoON, and L. C., Dicke, M. and others. 2005. Signal signature and transcriptome changes of Arabidopsis during pathogen and insect attack. Mol. Plant-Microbe Interact. 18:923-937.

DegenhardT, J. and, Gershenzon, J. 2000. Demonstration and characterization of (E)-nerolidol synthase from maize: a herbivore-inducible terpene synthase participating in (3E)-4,8dimethyl-1,3,7-nonatriene biosynthesis. Planta 210:815-822.

DICKE, M., and SABELIS, M. W. 1988. How plants obtain predatory mites as bodyguards. Neth. J. Zool. 38:148-165.

Ferry, A., Dugravot, S., Delattre, T., Christides, J. P., Auger, J., BAgneres, A. G., Poinsot, D., and Cortesero, A. M. 2007. Identification of a widespread monomolecular odor differentially attractive to several Delia radicum ground-dwelling predators in the field. J. Chem. Ecol. 33:2064-2077.
Gaquerel, E., Weinhold, A., and Baldwin, I. T. 2009. Molecular interactions between the specialist herbivore Manduca sexta (Lepidoptera, Sphigidae) and its natural host Nicotiana attenuata. VIII. An unbiased GCxGC-ToFMS analysis of the plant's elicited volatile emissions. Plant Physiol. 149:1408-1423.

Geervliet, J. B. F., Posthumus, M. A., Vet, L. E. M., and Dicke, M. 1997. Comparative analysis of headspace volatiles from different caterpillar-infested or uninfested food plants of Pieris species. J. Chem. Ecol. 23:2935-2954.

Geervliet, J. B. F., Verdel, M. S. W., Snellen, H., Schaub, J., DiCKE, M., and Vet, L. E. M. 2000. Coexistence and niche segregation by field populations of the parasitoids Cotesia glomerata and C. rubecula in the Netherlands: predicting field performance from laboratory data. Oecologia 124:55-63.

Geladi, P., and Kowalski, B. R. 1986. Partial Least-Squares Regression - a Tutorial. Anal. Chim. Acta 185:1-17.

Gols, R., Witjes, L. M. A., Van Loon, J. J. A., Posthumus, M. A., DicKe, M., and HaRVEY, J. A. 2008. The effect of direct and indirect defenses in two wild brassicaceous plant species on a specialist herbivore and its gregarious endoparasitoid. Entomol. Exp. Appl. 128:99-108.

Hopke, J., Donath, J., Blechert, S., and Boland, W. 1994. Herbivore induced volatiles: the emission of acyclic homoterpenes from leaves of Phaseolus lunatus and Zea mays can be triggered by a $\beta$-glucosidase and jasmonic acid. FEBS Lett. 352:146-150.

HOPKINS, R. J., VAN DAM, N. M., and VAN LOON, J. J. A. 2009. Role of glucosinolates in insect-plant relationships and multitrophic interactions. Annu.Rev. Entomol. 54:57-84.

IBRAhim, M. A., NisSinEN, A., and Holopainen, J. K. 2005. Response of Plutella xylostella and its parasitoid Cotesia plutellae to volatile compounds. J. Chem. Ecol. 31:1969-1984.

Jansen, J. J., Smit, S., Hoefsloot, H. C. J., and Smilde, A. K. 2010. The photographer and the greenhouse: How to analyse plant metabolomics data. Phytochemistry Anal. 21:48-60.

Jansen, J. J., Van Dam, N. M., Hoefsloot, and H. C. J., Smilde, A. K. 2009. Crossfit analysis: a novel method to characterize the dynamics of induced plant responses. BMC Informatics 10:425 http://www.biomedcentral.com/1471-2105/10/425

Kappers, I. F., Aharoni, A., VAN Herpen, T., LuCKerhoff, L. L. P., DiCKE, and M., BOUWMEESTER, H. J. 2005. Genetic engineering of terpenoid metabolism attracts, bodyguards to Arabidopsis. Science 309:2070-2072.

Kessler, A., and BALDWIN, I. T. 2001. Defensive function of herbivore-induced plant volatile emissions in nature. Science 291:2141-2144.

KugimiYA, S., Shimoda, T., TABATA, J., and TAKabayAShi, J. 2010. Present or past herbivory: A screening of volatiles released from Brassica rapa under caterpillar attacks as attractants for the solitary parasitoid, Cotesia vestalis. J. Chem. Ecol. 36:620-628.

Leitner, M., Kaiser, R., RASMUSSEN, M. O., Driguez, H., Boland, W., and MithofER, A. 2008. Microbial oligosaccharides differentially induce volatiles and signalling components in Medicago truncatula. Phytochemistry 69:2029-2040.

LOIVAMÄKI, M., HOlOPAINEN, J. K., and NerG, A. M. 2004. Chemical changes induced by methyl jasmonate in oilseed rape grown in the laboratory and in the field. J. Agric. Food Chem. 52:7607-7613.

Masters, G. J., Jones, T. H., and Rogers, M. 2001. Host-plant mediated effects of root herbivory on insect seed predators and their parasitoids. Oecologia 127:246-250.

MumM, R., and HiLKer, M. 2005. The significance of background odour for an egg parasitoid to detect plants with host eggs. Chem. Sens. 30:337-343.

Mumm, R., Posthumus, M. A., and Dicke, M. 2008. Significance of terpenoids in induced indirect plant defence against herbivorous arthropods. Plant Cell Environ. 31:575-585. 
Ozawa, R., Arimura, G., Takabayashi, J., Shimoda, T., and NISHIOKA, T. 2000. Involvement of jasmonate- and salicylaterelated signaling pathways for the production of specific herbivore-induced volatiles in plants. Plant Cell Physiol. 41:391-398.

Qiu, B.-L., Harvey, J. A., RaAijmakers, C. E., Vet, L. E. M., and VAN DAM, N. M. 2009. Non-linear effects of plant root and shoot jasmonic acid application on the performance of Pieris brassicae and its parasitoid Cotesia glomerata. Func. Ecol. 23:496-505.

Rasmann, S., Kollner, T. G., Degenhardt, J., Hiltpold, I., Toepfer, S., Kuhlmann, U., Gershenzon, J., and Turlings, T. C. J. 2005. Recruitment of entomopathogenic nematodes by insect-damaged maize roots. Nature 434:732-737.

RASMANN, S., and TuRLingS, T. C. J. 2007. Simultaneous feeding by aboveground and belowground herbivores attenuates plantmediated attraction of their respective natural enemies. Ecol. Lett. 10:926-936.

Rohloff, J., and Bones, A. M. 2005. Volatile profiling of Arabidopsis thaliana-Putative olfactory compounds in plant communication. Phytochemistry 66:1941.

Schnee, C., Kollner, T. G., Held, M., Turlings, T. C. J., Gershenzon, J., Degenhardt, J. 2006. The products of a single maize sesquiterpene synthase form a volatile defense signal that attracts natural enemies of maize herbivores. Proc. Natl. Acad. Sci. USA 103:1129-1134.

Schuman, M. C., Heinzel, N., Gaquerel, E., Svatos, A., and BALDWIN, I. T. 2009. Polymorphism in jasmonate signaling partially accounts for the variety of volatiles produced by Nicotiana attenuata plants in a native population. New. Phytol. 183:1134-1148.

Smid, H. A., Van Loon, J. J. A., Posthumus, M. A., and Vet, L. E. M. 2002. GC-EAG-analysis of volatiles from Brussels sprouts plants damaged by two species of Pieris caterpillars: olfactory receptive range of a specialist and a generalist parasitoid wasp species. Chemoecology 12:169-176.

Smid, H. M., Wang, G. H., Bukovinszky, T., Steidle, J. L. M., Bleeker, M. A. K., VAN LoON, J. J. A., and Vet, L. E. M. 2007. Species-specific acquisition and consolidation of long-term memory in parasitic wasps. Proc. R. Soc. B Biol. Sci. 274:1539-1546.
SoKal, R. R., and RoHLF, J. F. 1995. Biometry: The Principles and Practice of Statistics in Biological Research. New York: Freeman and Co. $850 \mathrm{p}$.

Soler, R., Bezemer, T. M., Van Der Putten, W. H., Vet, L. E. M., and HARVEY, J. A. 2005. Root herbivore effects on an aboveground herbivore, parasitoid and hyperparasitoid performance via changes in plant quality. J. Animal Ecol. 74:1121-1130.

Soler, R., Harvey, J. A., Kamp, A. F. D., Vet, L. E. M., VAN Der Putten, W. H., Van Dam, N. M., Stuefer, J. F., Gols, R., HordiJK, C. A., and BEZEMER,T.. 2007. Root herbivores influence the behaviour of an aboveground parasitoid through changes in plant-volatile signals. Oikos 116:367-376.

THOLL, D. 2006. Terpene synthases and the regulation, diversity and biological roles of terpene metabolism. Curr. Op. Plant Biol. 9:297-304.

Turlings, T. C. J., Tumlinson, J. H., and Lewis, W. J. 1990. Exploitation of herbivore-induced plant odors by host-seeking parasitic wasps. Science 250:1251-1253.

VAN DAM, N. M., and POPPY, G. M. 2008. Why plant volatile analysis needs bioinformatics - detecting signal from noise in increasingly complex profiles. Plant Biol. 10:29-37.

VAn Dam, N. M., WitJes, L., and Svatos, A. 2004. Interactions between aboveground and belowground induction of glucosinolates in two wild Brassica species. New. Phytol. 161:801-810.

Van Poecke, R. M. P., Posthumus, M. A., and Dicke, M. 2001. Herbivore-induced volatile production by Arabidopsis thaliana leads to attraction of the parasitoid Cotesia rubecula: Chemical, behavioral, and gene-expression analysis. J. Chem. Ecol. 27:1911-1928.

VET, L. E. M. 1999. Evolutionary aspects of plant-carnivore interactions. pp 3-20.in: CHADWICK, D. J., GOODE, J., (eds.). Insect-Plant Interactions and Induced Plant Defence. Sussex, UK: John Wiley \& Sons Ltd.

Vet, L. E. M., and Groenewold, A. W. 1990. Semiochemicals and learning in parasitoids. J. Chem. Ecol. 16:3119-3135.

Vet, L. E. M., Lewis, W. J., and CARdÉ, R. T. 1995. Parasitoid foraging and learning pp 65-101 in: BELL W., CARDÉ, R. T., (eds.). Chemical Ecology of Insects, 2nd edition. London: Chapman and Hall. 